\title{
Drug interaction alerts in software - what do general practitioners and pharmacists want?
}

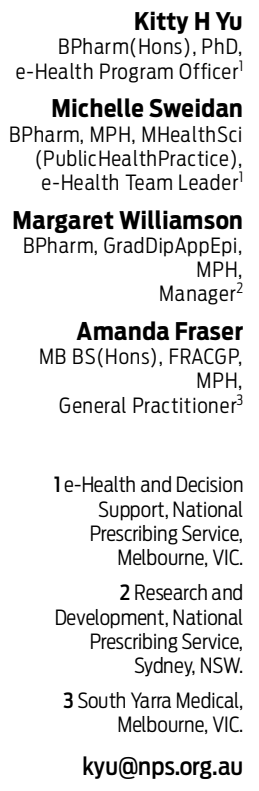

MJA 2011; 195: 676-680 doi: 10.5694/mjall.10206

\section{.}

rug interactions can be dangerous and at times lethal., ${ }^{1,2}$ Drug interaction alert messages in clinical software may help clinicians decide whether certain drug combinations can be used safely. Most clinical software systems include drug interaction decision support; however, they use different reference sources and they implement and present drug interaction information in different ways. ${ }^{3,4}$ Some clinicians have said that they are bombarded with too many alerts that they find annoying and that the information provided is sometimes irrelevant or unhelpful. ${ }^{5-7}$ Despite this, general practitioners and pharmacists generally believe the benefits are sufficiently important for them not to switch off alerts. 8,9

There are currently no standards or guidelines in Australia pertaining to the quality or suitability of drug interaction knowledge bases for decision support, and little guidance on how such knowledge bases should be implemented in clinical software (eg, how or when information is displayed to users). In addition, a recent study found that drug interaction decision support in commonly used prescribing and dispensing systems in Australia has significant shortcomings; namely, variation between systems, low specificity of alerts in some systems, and a lack of information on clinical effects and management advice. ${ }^{4}$ An expert panel made recommendations for improving the content and format of drug interaction alerts. ${ }^{4}$

We aimed to find out whether Australian GPs and pharmacists agreed with these recommendations and to explore their preferences in relation to the content, format and usability of drug interaction alerts. While there are numerous barriers and facilitators to the uptake of decision support, ${ }^{10}$ we were particularly interested in the format of drug interaction alerts because format can influence how users respond to and use decision support ${ }^{11,12}$ and research in this area

Objective: To explore Australian general practitioners' and pharmacists'
preferences in relation to content, format and usability of drug interaction preferences in relation to content, format and
alerts in prescribing and dispensing software.

Design, participants and setting: Surveys that sought opinions on drug interaction decision support were mailed to a random sample of GPs and community pharmacists (1000 of each) in June 2010.

Main outcome measures: Usefulness of various components of drug interaction information; preferred format of drug interaction alerts; levels of agreement on the value of various usability features; aspects of drug interaction decision support users would most like to change.

Results: Surveys were returned by 219 GPs and 170 pharmacists. Of the 191 GPs and 138 pharmacists included in the analysis, the vast majority considered severity, clinical effects and management advice to be mostly or sometimes useful in drug interaction alerts. The most popular drug interaction alert format - favoured by 131 GPs (69\%) and 115 pharmacists (83\%) - was one with headings and one or two succinct bullet points under each. The vast majority of respondents also wanted to be able to differentiate drug interaction alerts by severity, and a majority agreed that it should be made more difficult to override alerts for severe interactions and that it should be mandatory to provide a reason for doing so.

Conclusions: GPs and pharmacists want drug interaction alert information to be relevant, useful, concise, and easy to read and comprehend. Software vendors and knowledge providers could improve drug interaction decision support by making changes to the content and format of drug interaction alerts according to our recommendations.

is limited. We also aimed to develop recommendations for software vendors and knowledge providers that would lead to more useful and acceptable decision support for GPs and pharmacists.

\section{Methods}

Ethics approval for this study was granted by the Royal Australian College of General Practitioners National Research and Evaluation Ethics Committee.

\section{Survey development}

A postal survey for GPs and community pharmacists was developed, with the content informed by expert panel recommendations $s^{4}$ and a literature review (conducted in February 2010) on drug interaction decision support. The key survey questions are shown in Box 1; demographic information was also collected. The survey questions were the same for GPs and pharmacists, apart from the eligibility questions (which were worded differently to reflect prescribing and dispensing processes). The survey was piloted for face validity by two GPs and two community pharmacists.

\section{Selection of participants}

GPs and pharmacists were eligible to participate in the study if they currently practised, used a computer to prescribe or perform the data entry step of dispensing, and had drug interaction alerts switched on in their prescribing or dispensing software. A standard sample-size calculator for prevalence surveys ${ }^{13}$ was used to generate required sample-sizes of 244 GPs and 242 pharmacists, with four assumptions: $80 \%$ of respondents would agree that four key components of information in a drug interaction alert ${ }^{4}$ are useful; Australian GP and community pharmacist populations of 24000 and 13000, respectively; a 95\% confidence interval; and 
$5 \%$ precision. We multiplied the required sample sizes by four, based on an expected response rate of $25 \%$, and rounded these numbers up to 1000. The postal survey was sent to 1000 GPs and 1000 pharmacists who had been randomly selected from a comprehensive database of health professionals practising in Australia (AMPCo Direct).

The survey was mailed out in June 2010, non-respondents were sent a reminder 3 weeks later and the survey was closed in July 2010. Data from returned surveys were collated and de-identified. Quantitative data were machine-scanned and $20 \%$ of these data were cross-checked against returned surveys for accuracy by one of us (KHY). Free-text responses were entered into spreadsheets by administrative staff, then reviewed by one of us (KHY) to ensure that handwriting was accurately interpreted.

\section{Data analysis}

Quantitative data were analysed with Microsoft Excel (Microsoft Corporation, Redmond, Wash, USA) and SPSS v18 (SPSS Inc, Chicago, Ill, USA) using descriptive statistics. Free-text responses were analysed qualitatively by theme.

\section{Results}

Surveys were returned by 219 GPs and 170 pharmacists (22\% and 17\% response rates, respectively). After excluding surveys from ineligible respondents, 191 GP surveys and 138 pharmacist surveys were analysed. Characteristics of respondents included in the analysis are shown in Box 2. Cross-checking of machinescanned data showed a $0.17 \%$ error rate.

\section{Components of information}

GPs and pharmacists believed that the most useful components of information in drug interaction alerts were severity, clinical effects and management advice (Box 3). Also useful but not to the same extent - were information on timeframe, mechanism, the date of the last update of the information source and access to a list of reference sources. Some GPs and pharmacists indicated that information on the frequency or likelihood of adverse effects arising from drug interactions would also be useful.

Respondents provided over 100 free-text complaints about irrelevant or minor alerts. They found alerts for the following to be unhelpful: theoretical interactions; dosage forms that do not interact (eg, creams, inhalers); individual drugs within a class that do not interact (eg, not all calcium channel blockers have the same interactions); drugs routinely used together; drugs no longer being taken by the patient; and duplicate alerts (eg, when different strengths of warfarin are prescribed).

\section{Format of drug interaction alerts}

Most GPs and pharmacists preferred drug interaction alerts to be in the "headings + bullets" format (Box 4). Reasons provided were that this format is clear, concise, easy to scan through or read, and easy to navigate. Some respondents indicated that they wanted the alerts in their systems to better draw their attention - for example, larger pop-up boxes and text size, and more colour and visual effects. Some also wanted changes that would reduce the need for scrolling and changes in the amount of information the drug interaction alert displayed initially, to aid more rapid comprehension of information.

\section{Preferences for usability features}

The levels of agreement among respondents on the value of various usability features in drug interaction decision support are shown in Box 5 . Several GPs stated that when there are multiple drug interaction alerts for a patient, those with the most severe warning should appear first. Some respondents suggested ways to customise drug interaction alerts, including suppression of minor and moderate alerts, alerts on repeat prescriptions and alerts that have appeared more than once or twice for a patient.

\section{Three things to change}

The three aspects of drug interaction decision support GPs and pharmacists would most like to change in their systems were: make the drug interaction alerts more relevant to practice; improve the format and presentation of drug interaction alerts;
1 Key survey questions on content, format and usability of drug interaction decision support

\section{Content}

Previous NPS work has indicated that components of information may be useful in drug interaction alert messages. How useful do you think each of these components is to your clinical practice? (Mostly useful, sometimes useful, not useful, or not sure)

- Clinical effects

- Management advice

- Time frame

- Mechanism

How useful would the following additional components of information be? (Mostly useful, sometimes useful, not useful, or not sure)

- Severity of the interaction

- Access to a list of reference sources

- Date that information was last updated

- Others (please specify)

\section{Format}

The following table shows a drug interaction alert presented in three different formats.* Please indicate one preferred format and the reason(s) for your choice. (Select one option or "I do not like any of these"; free text)

\section{Usability}

Do you disagree or agree that the following features of drug interaction alerts would be desirable in prescribing or dispensing software? (Strongly disagree, disagree, agree, strongly agree, or not sure)

- Ability to customise drug interaction alerts

- Links to more detailed information

- Ability to differentiate drug interaction alerts from other types of warning messages

- Ability to differentiate drug interaction alerts by severity

- Make it harder to override alerts for severe interactions

- Make it mandatory for users to provide a reason when

overriding an alert for a severe interaction

\section{Three things to change}

What are the three most important things that you would change about the drug interaction alerts in your prescribing or dispensing system? (Free text)

NPS = National Prescribing Service. $*$ Examples of the three formats are shown in Box 4.

2 Characteristics of general practitioners and pharmacists included in the analysis*

\begin{tabular}{lcc} 
& $\begin{array}{c}\text { GPs } \\
(n=191)\end{array}$ & $\begin{array}{c}\text { Pharmacists } \\
(n=138)\end{array}$ \\
\hline Men & $116(61 \%)$ & $69(50 \%)$ \\
Age & $15(8 \%)$ & $55(40 \%)$ \\
$<35$ years & $120(63 \%)$ & $60(43 \%)$ \\
$35-54$ years & $54(28 \%)$ & $23(17 \%)$ \\
$>54$ years & $2(1 \%)$ & 0 \\
Not answered & $21.4(11.0)$ & $18.4(12.9)$ \\
Average years practised (SD) & $35.6(13.2)$ & $38.1(10.4)$ \\
Average hours worked per week (SD) & \\
Location of main place of practice & $58(30 \%)$ & $48(35 \%)$ \\
New South Wales & $43(23 \%)$ & $31(22 \%)$ \\
Victoria & $32(17 \%)$ & $28(20 \%)$ \\
Queensland & $24(13 \%)$ & $18(13 \%)$ \\
Western Australia & $17(9 \%)$ & $8(6 \%)$ \\
South Australia & $8(4 \%)$ & $5(4 \%)$ \\
Tasmania & $4(2 \%)$ & 0 \\
Australian Capital Territory & $1(1 \%)$ & 0 \\
Northern Territory & $4(2 \%)$ & 0 \\
Not answered & & \\
\hline
\end{tabular}

*Data are number (\%) unless otherwise indicated. 
3 Proportions of general practitioners and pharmacists who considered various components of drug interaction alerts to be mostly or sometimes useful*
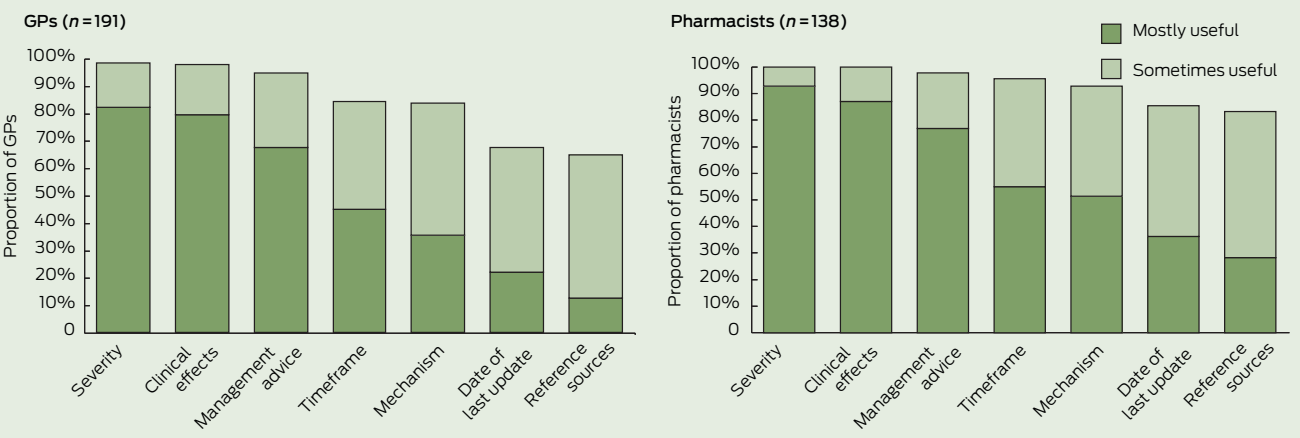

*Missing data and data for the responses "not useful" and "not sure" are not shown.

and include information about drug interaction severity and differentiate drug interaction alerts by severity.

\section{Respondent comments}

Examples of respondents' comments on various aspects of drug interaction decision support are shown in Box 6 .

\section{Discussion}

In this study, GPs and pharmacists indicated what is important to them in relation to drug interaction decision support in clinical software, including components of information they would find useful, the best way to present information, and usability features that they would value. They concurred with previous recommendations ${ }^{4}$ — in particular, almost all GPs and pharmacists wanted information on clinical effects and management advice, which is not consistently available in commonly used general practice and pharmacy software systems.

GPs and pharmacists also wanted information on severity, including differentiation of drug interaction alerts by severity. Information on the severity of an interaction has been used to prioritise and increase the acceptance of alerts. ${ }^{14}$ However, there has been no comprehensive assessment of the validity of severity ratings ${ }^{15}$ and studies have shown a high degree of discordance between different reference sources in the severity ratings assigned to drug interactions. ${ }^{15,16}$ There are a number of reasons for this, including a lack of good epidemiological evidence for drug interactions and the use of variable terminology and classification systems. ${ }^{15,17}$ Given the overwhelming support from GPs and pharmacists for severity information to be available, this should be explored further and efforts should be made to ensure that severity ratings are consistent and reliable. In addition, current users of drug interaction alerts should be made aware of the limitations of severity ratings.

GPs and pharmacists perceived some drug interaction alerts to be unhelpful or irrelevant to decision making. Reasons for this included irrelevant content and alerts for clinically unimportant interactions. A lack of sophistication in decision support systems can also be a factor; for example, systems may not recognise drugs that a patient is no longer taking or that have already been prescribed in another strength. While there are valid reasons for intentionally overriding alerts, ${ }^{18}$ clinicians may also ignore alerts because of alert fatigue. ${ }^{19,20}$ This can have serious implications if critical drug interaction alerts are inadvertently bypassed.

Respondents in our study indicated a preference for presentation of drug interaction information using headings (eg, "clinical effects" and "management") with one or two succinct bullet points of information under each. Similarly, previous work by the National Prescribing Service (unpublished data) found that providing information to GPs in this format facilitated scanning of information, and that headings drew GPs' attention to the information of greatest interest to them. Very few respondents preferred the paragraph format, but this is the format currently used for drug interaction alert messages in many software systems.

\section{General practitioners' and pharmacists' preferences for drug interaction alert formats*}

\section{Example: methotrexate and trimethoprim}

$\begin{array}{ll}\text { "Heading + bullets" format } & \text { "Bullets" format } \\ \text { Effects } & \text { Combination may cause life-threatening } \\ \text { Combination may cause life-threatening } & \text { bone marrow suppression (in weeks to } \\ \text { bone marrow suppression } & \text { months) } \\ \text { Timeframe } & \text { Avoid combination if possible } \\ \text { Weeks to months } & \text { If concurrent use is necessary, monitor } \\ \text { Management } & \text { blood count closely } \\ \text { Avoid combination if possible } & \text { Mechanism is unclear; may be additive } \\ \text { If concurrent use is necessary, } & \text { antifolate effects or decreased renal } \\ \text { monitor blood count closely } & \text { clearance of methotrexate } \\ \text { Mechanism } & \\ \text { Unclear; may be additive antifolate } & \\ \text { effects or decreased renal clearance of } & \\ \text { methotrexate } & \\ \text { Preferred by: } & \text { Preferred by: } \\ 131 \text { GPs (69\%) } & 45 \mathrm{GPs}(24 \%) \\ 115 \text { pharmacists (83\%) } & 21 \text { pharmacists (15\%) }\end{array}$

\section{“Paragraph" format}

Combination may cause life-threatening bone marrow suppression (in weeks to months). Avoid combination if possible, or if concurrent use is necessary, monitor blood count closely. Mechanism is unclear; may be additive antifolate effects or decreased renal clearance of methotrexate.

* One GP and two pharmacists did not like any of the formats; seven GPs did not respond to the question.
Preferred by: 7 GPs (4\%) 0 pharmacists


More than $80 \%$ of GPs and pharmacists in our study thought it should be more difficult for users to override severe interaction alerts. One way to do so is to make it mandatory for users to provide a reason when they override a severe interaction alert. Users may find it useful to record their actions as a way of reminding themselves or informing others of their decision and to justify their reason for overriding an alert. About half the respondents agreed or strongly agreed that users should be able to customise drug interaction alerts (eg, suppress an alert if the user is already familiar with it). Some users may welcome the option of tailoring which drug interactions generate alerts, while the fear of missing potentially important interactions may deter others. ${ }^{5,9}$

Our findings support those of others. It has previously been shown that clinicians want information on management advice and severity of interactions. $^{21-23}$ Many clinicians would like to be able to differentiate drug interaction alerts by severity, increase the difficulty of overriding alerts for severe interactions, and make it mandatory for users to provide a reason for doing so. ${ }^{7,9,23,24}$ Clinicians' frustration with irrelevant or unhelpful alerts has also been reported. ${ }^{6,7,25}$

Our findings on the presentation of alert messages aligns with recent research showing that the way information is displayed to users affects workflow and acceptance of decision support. ${ }^{11,12}$ In an observational study of users' responses to medication alerts, it was found that a key barrier to workflow and decision making was poor screen display. ${ }^{11}$ Another study showed a strong correlation between the quality of the display and whether an alert was accepted or not. ${ }^{12}$ Our study highlighted that changes to the format of an alert message can improve readability and navigation without altering the content.

Our study had some limitations. The response rate was lower than expected, limiting generalisability. Nevertheless, the opinions of the respondents provide valuable guidance for developing decision support systems for Australian health professionals - to our knowledge, this is the first Australian study on this topic to be published. Also, while there was a high level of agreement among
5 Levels of agreement among general practitioners and pharmacists on the value of various drug interaction alert usability features*

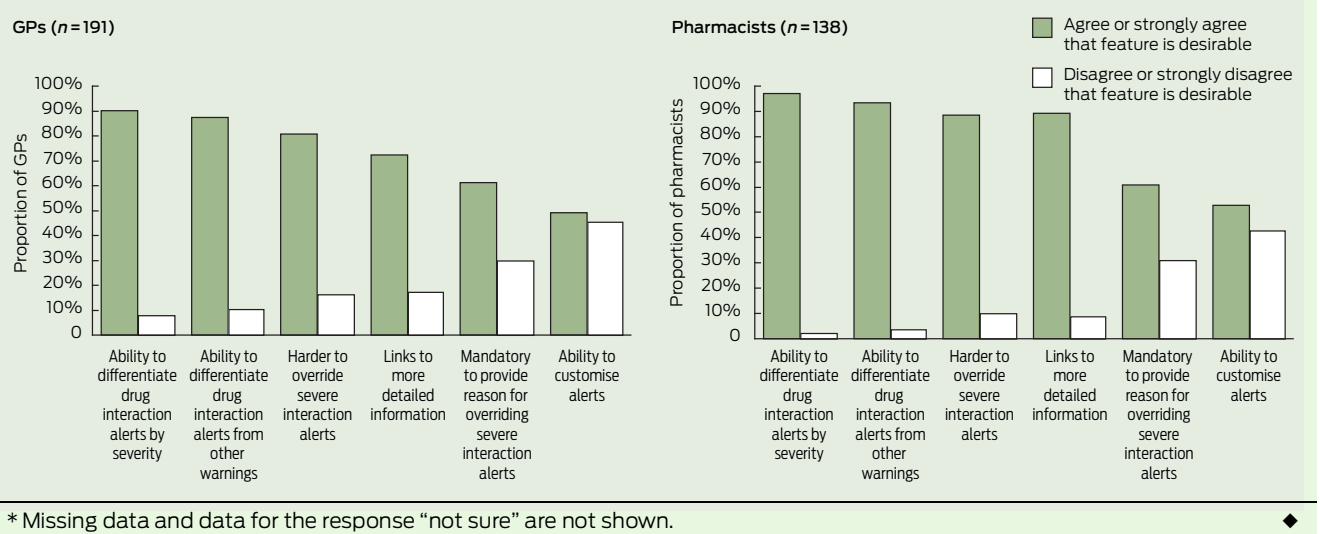

\section{General practitioners' and pharmacists' comments on various aspects of drug interaction decision support \\ Content}

"Have information on the management [of drug interactions] or [offer] alternative medicines that won't interact." (GP) "[Include information on] the percentage risk of a drug interaction causing problems for the patient." (GP)

Format

"Point form with relevant headings [makes it] much easier to find relevant information ..." (Pharmacist)

"Keep it brief and to the point. Don't have time to read large amounts of information." (Pharmacist)

\section{Severity}

"Need ... severe life-threatening interaction at top of list ... not placed in the middle of two possible theoretical interactions and all given the same formatting." (GP)

"It is a trap to overlook an interaction that appears to be common and/or minor but if there are options to customise such alerts, that would be very helpful." (Pharmacist)

\section{Relevance}

"Get rid of ridiculous minor interactions (eg, Ventolin for diabetes etc). There are heaps of these and they are 'white noise'. [They] could potentially stop you from noticing more serious interactions." (GP)

"Remove theoretical unimportant alerts - they are a distraction and devalue significant alerts." (GP)

Other

"[Drug] interaction alerts should be the same for all software." (Pharmacist) respondents on many factors, those who did not respond may have had different views.

This study offers an Australian perspective on the issues of content, format and usability of drug interaction decision support and provides evi- dence of shortcomings that drug interaction knowledge providers and software vendors need to address for users. Drug interaction alerts could be improved by changes to content and format according to our recommendations (Box 7).

\section{Recommendations for software vendors and knowledge providers to improve drug interaction decision} support, and areas for further investigation

\section{Recommendations}

- Content: Drug interaction messages should include information on clinical effects, management advice, timeframe and mechanism, where this information is known. Inclusion of information on severity requires further investigation.

- Format: Information should be presented in an easy to read format that includes headings (eg, "clinical effects", "management advice") and bullet points.

- Relevance of information: Drug interaction knowledge bases should include only clinically significant drug interactions. They should be structured so that warnings can be provided for individual drugs (where not all drugs in a class interact in the same way) and for the prescribed route of administration (eg, oral formulations but not creams and ointments, where applicable).

- Reduction of alert fatigue: There should not be warnings for drugs that are not currently used by the patient, nor duplicate warnings where one drug is prescribed in different strengths (eg, warfarin).

\section{Areas for further investigation}

- Further work is required to ensure that severity ratings are reliable for users. Health professionals should be aware that severity is context-dependent and that severity ratings can vary markedly between different prescribing and dispensing systems; caution is needed when interpreting information on severity.

- Customisation of drug interaction decision support by users may be useful but more research on how this can be done safely in practice is needed.

- Participants in this study believed that it should be made harder for users to override severe drug interaction alerts and that it should be mandatory for users to provide a reason when they do so. How this is best implemented requires further investigation. 
Acknowledgements: We thank James Reeve, Jonathan Dartnell and Malcolm Gillies (National Prescribing Service) for helpful comments in preparation of this manuscript.

Competing interests: No relevant disclosures.

Received 24 Feb 2011, accepted 6 Oct 2011.

1 New South Wales Department of Health. Safety notice: 011/09. Allopurinol and azathioprine. 7 May 2009. http://www.health.nsw.gov.au/ resources/quality/sabs/pdf/sn20090507.pdf (accessed Nov 2011).

2 Pilgrim JP, Gerostamoulos D, Drummer OH. Deaths involving contraindicated and inappropriate combinations of serotonergic drugs. Int J Legal Med 2011; 125: 803-815.

3 Saverno KR, Hines LE, Warholak TL, et al. Ability of pharmacy clinical decision-support software to alert users about clinically important drugdrug interactions. J Am Med Inform Assoc 2010; 18: 32-37.

4 Sweidan M, Reeve JF, Brien JE, et al. Quality of drug interaction alerts in prescribing and dispensing software. Med J Aust 2009; 190 : 251-254.

5 Ahearn MD, Kerr SJ. General practitioners' perceptions of the pharmaceutical decisionsupport tools in their prescribing software. Med J Aust 2003; 179: 34-37.

6 Lapane KL, Waring ME, Schneider KL, et al. A mixed method study of the merits of e-prescribing drug alerts in primary care. J Gen Intern Med 2008; 23: 442-446.

7 Magnus D, Rodgers S, Avery AJ. GPs' views on computerized drug interaction alerts: questionnaire survey. J Clin Pharm Ther 2002; 27: 377-382.
8 Glassman PA, Belperio P, Simon B, et al. Exposure to automated drug alerts over time: effects on clinicians' knowledge and perceptions. Med Care 2006; 44: 250-256.

9 Weingart SN, Massagli M, Cyrulik A, et al. Assessing the value of electronic prescribing in ambulatory care: a focus group study. Int J Med Inform 2009; 78: 571-578.

10 Moxey A, Robertson J, Newby D, et al. Computerized clinical decision support for prescribing: provision does not guarantee uptake. J Am Med Inform Assoc 2010; 17: 25-33.

11 Russ AL, Zillich AJ, McManus MS, et al. A human factors investigation of medication alerts: barriers to prescriber decision-making and clinical workflow. AMIA Annu Symp Proc 2009; 2009: 548-552.

12 Seidling HM, Phansalkar S, Seger DL, et al. Factors influencing alert acceptance: a nove approach for predicting the success of clinical decision support. J Am Med Inform Assoc 2011; 18: 479-484

13 Glaziou P. Sample size for a prevalence survey, with finite population correction. http:// sampsize.sourceforge.net/iface/index.htm (accessed Mar 2010).

14 Paterno MD, Maviglia SM, Gorman PN, et al. Tiering drug-drug interaction alerts by severity increases compliance rates. J Am Med Inform Assoc 2009; 16: 40-46.

15 Vitry Al. Comparative assessment of four drug interaction compendia. Br J Clin Pharmacol 2007; 63: 709-714.

16 Wang LM, Wong M, Lightwood JM, et al. Black box warning contraindicated comedications: concordance among three major drug interaction screening programs. Ann Pharmacother 2010; 44 28-34.
17 Abarca J, Malone DC, Armstrong EP, et al. Concordance of severity ratings provided in four drug interaction compendia. J Am Pharm Assoc (2003) 2004; 44: 136-141.

18 Weingart SN, Toth M, Sands DZ, et al. Physicians' decisions to override computerized drug alerts in primary care. Arch Intern Med 2003; 163: 26252631

19 Weingart SN, Simchowitz B, Shiman L, et al. Clinicians' assessments of electronic medication safety alerts in ambulatory care. Arch Intern Med 2009; 169: 1627-1632.

20 Cash JJ. Alert fatigue. Am J Health Syst Pharm 2009; 66: 2098-2101.

21 Bergk V, Gasse C, Schnell R, et al. Requirements for a successful implementation of drug interaction information systems in general practice: results of a questionnaire survey in Germany. Eur J Clin Pharmacol 2004; 60: 595-602.

22 Indermitte J, Erba L, Beutler M, et al. Management of potential drug interactions in community pharmacies: a questionnaire-based survey in Switzerland. Eur J Clin Pharmacol 2007; 63: 297-305.

23 Ko Y, Abarca J, Malone DC, et al. Practitioners' views on computerized drug-drug interaction alerts in the VA system. J Am Med Inform Assoc 2007; 14: 56-64.

24 Murphy JE, Forrey RA, Desiraju U. Community pharmacists' responses to drug-drug interaction alerts. Am J Health Syst Pharm 2004; 61: 14841487.

25 Abarca J, Malone DC, Skrepnek GH, et al. Community pharmacy managers' perception of computerized drug-drug interaction alerts. J Am Pharm Assoc (2003) 2006; 46: 148-153. 